\title{
THE POWER SUPPLY SYSTEMS FOR ELLIPTICAL MULTIPOLE WIGGLERS
}

\author{
A.S. Medvedko, Yu.A. Evtushenko, B.A. Dovgenko, S.P. Petrov, V.F. Veremeenko, \\ Budker Institute of Nuclear Physics, 630090, Novosibirsk, Russia \\ E. A. Medvedko, \\ Argonne National Laboratory, Argonne, Ill, 60439, USA, (now at SLAC) \\ Om Vir Singh, \\ Brookhaven National Laboratory, Upton, NY. 11973, USA
}

\section{Abstract}

The Elliptical Multipole Wiggler (EMW; 3.4 meter-long) and its Prototype $(0.8 \mathrm{~m})$ had been built by the joint team of INP (Novosibirsk, Russia), ANL (Argonne, USA), and BNL (Upton, USA). These Wigglers are supplied by the DC or trapezoidal shape AC current with variable frequency. Current is in 200 A to 1200 A range. Two different PSs were developed: for Prototype - with the frequency range up to $100 \mathrm{~Hz}$, for EMW - up to $10 \mathrm{~Hz}$. The PSs have the original circuit for current polarity forced switching. These circuits allow us to get the rise/fall time twenty times faster than that determined by magnet time constant: it became $1.5 \mathrm{~ms}$ for Prototype and $5 \mathrm{~ms}$ for EMW. Top of the pulses have the flatness better than 0.01 , current difference for both polarities better than 0.001. Prototype is under the operation at the NSLS (BNL) during two years. EMW has been installed, tested and first beam for users would be taken out soon at the APS (ANL).

\section{INTRODUCTION}

The EMW generates elliptically polarized photons in the energy range of $0.1-10 \mathrm{keV}$ with $\mathrm{AC}$ modulation of polarization helixity. This type of wigglers will make it possible to detect the very weak signatures of circular dichroism and other effects associated with right- left- handedness of some physical systems [1]. To increase the measurement accuracy of the asymmetry between the effect of left and right circularly polarized radiation, the modulation frequency should be located in the range with the minimum spectrum power density of closed orbit noise. From this point of view, it was determined that the preferable modulation frequencies are: 0 ; Fmod ; $10 \mathrm{~Hz}$ and $\mathrm{F} \sim 100 \mathrm{~Hz}$. The EMW includes a vertical hybrid permanent magnet structure and a horizontal electromagnet structure. Prototype wiggler ( $0.8 \mathrm{~m}$ length, $27 \mathrm{~mm}$ aperture $)$ has a magnetic field period of $160 \mathrm{~mm}$ with seven poles. Field value is of $0.83 \mathrm{~T}$ for hybrid structure and of $0.12 \mathrm{~T}$ - for electromagnet [2]. The EMW has 36 poles with period of $80 \mathrm{~mm}$ and the aperture of $71 \mathrm{~mm}$ with closely the same field values. The core of electromagnet is fabricated from the laminated iron to operate with a switching frequency up to $100 \mathrm{~Hz}$.

\section{MAGNET PARAMETERS}

The electromagnet structures of EMW and of Prototype are the inductive loads with the Resistance $5.6 \mathrm{mOhm}$ (3 mOhm, Prototype) and Inductance $500 \mu \mathrm{H}(100 \mu \mathrm{H})$, operating current range 200-1200 A. So the electromagnet structure of EMW can store $360 \mathrm{~J}$ of energy $(75 \mathrm{~J})$. Load time constant is approximately $90 \mathrm{msec}(33 \mathrm{msec})$. It was desired to have changeable polarity of current with the trapezoidal in-time shape of current with operating frequency range from zero up to $10 \mathrm{~Hz}$ for EMW and up to $100 \mathrm{~Hz}$ for the Prototype. The top part of the current need to have the flatness better than $1 \%$, current difference for both polarities better than $0.1 \%$.

\section{POWER SUPPLY OPERATION PRINCIPLE}

The Function Diagram of the PS is presented at the Fig.1. The input $\mathrm{AC}$ power is going to the primary windings of the voltage reducing Transformer. The secondary Transformer coils are connected to the gate controlled Thyristor Rectifier Bridge with RLC-filter. Transformer, Rectifier, and RLC-filter form the "V-1000 Thyristor Rectifier".

The V-1000 output DC current with variable value from 200 A up to $1200 \mathrm{~A}$ is going through the choke L1, Direct Current Current Transformer DCCT-1 and Commutating choke L 2 to input diagonal of the Thyristor Bridge-Inverter SW1-SW4. Its output diagonal is connected to the EMW Electromagnet Coil with Capacitor $\mathrm{C} 1$ in parallel. Commutating choke has two secondary coils, connected into both current buses, and one primary joined with Commutator's output.

Let us say that the Bridge-Inverter has the initial position with SW1 and SW4 Thyristors are switched on (into the conducting mode), but SW2 and SW3 are switched off (into the isolation mode). The Electromagnet current direction is shown at the Fig. 1 by left arrow. For current polarity changing we need to switch off the SW1, SW4 and to switch on the SW2, SW3 Thyristors. The Commutator with choke L2 is used for realizing this process. Time Diagrams of switching processes are presented at the Fig.2.

The Commutator Thyristor SWcm, being driven by Clock external signal via Switch Controller, connects the Capacitor $\mathrm{Ccm}$ to the primary coil of choke L2. This Capacitor had been charged preliminary. The Capacitor discharging process applies the pulse with the reversed 


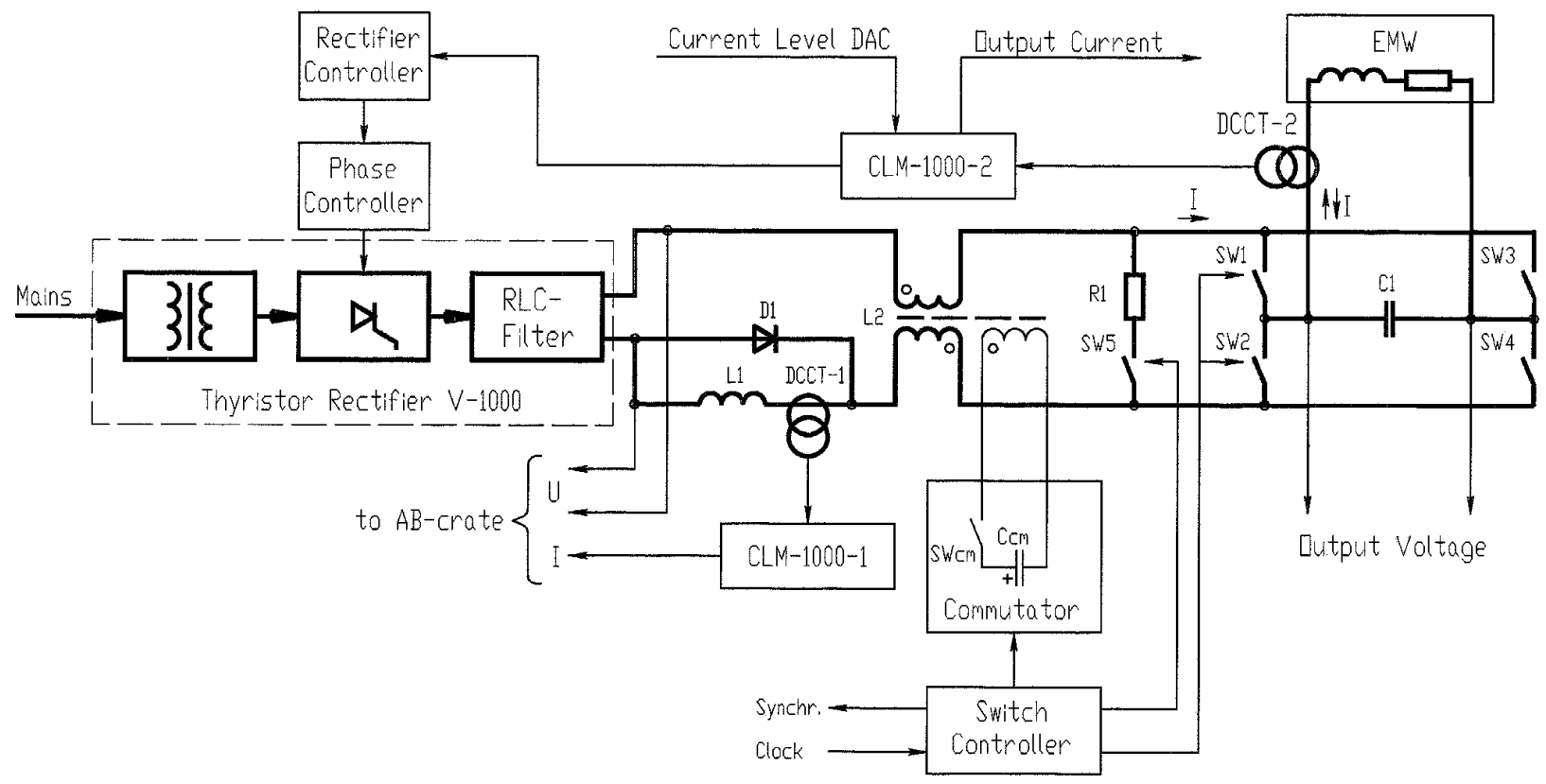

Figure 1: EMW function diagram.

(closing) polarity to input diagonal of the Bridge-Inverter. This time interval corresponds to positive polarity pulse at the time diagram. This pulse cause breaking current through the Switches SW1 and SW4. It need approximately $15 \mu \mathrm{sec}$ for Thyristors to switch off the current. So, after this time duration the Bridge-Inverter became switched off. The resonant circuit formed with Electromagnet coils and Capacitor $\mathrm{C} 1$ became isolated, so the energy stored in the Electromagnet magnetic field begins to recharge the capacitor and after that - to reverse the current polarity. It needs approximately half of free oscillating period (5 to $6 \mathrm{msec}$ for EMW and $1.5 \mathrm{msec}$ for Prototype) of this circuit for Electromagnet current polarity reversing. One can see that due to this oscillating process the voltage on the resonant circuit increases up to 300-350 Volts for the $1000 \mathrm{~A}$ input current (DC voltage on the coil is approximately $6 \mathrm{~V}$ ). This method of current switching is some time named like "Forced Switching".

The isolated ideal LC circuit has no energy dissipation so it reverses the polarity of $100 \%$ of Current. Real RLC circuit with Electromagnet coil of EMW and capacitor C1 have quality factor:

$$
Q=(\sqrt{L / C}) / R \approx 10,
$$

that determines the relatively noticeable level of the energy dissipation during the half of free oscillation period. As a result, the coil reversed current value at the end of this time interval (Fig.2) became equal to:

$$
\begin{gathered}
I_{\text {rev }}=I_{\text {ini }}(1-\delta / 2), \text { where } \\
\delta=\pi R \sqrt{C / L} .
\end{gathered}
$$

In our case we had got $I_{\text {rev }} \approx 0.85 I_{i n i}$ for EMW and $0.9 I_{i n i}$ for the Prototype; $I_{i n i}-$ initial Current value.

During the transient process and all time later the Switch Controller generates the pulses to fire the Switches SW2 and SW3. These Switches come into conducting mode when the voltage applied to them became in conducting direction that means the end of free oscillation process. This moment determines the switching on of the negative feedback loop that helps to output current to get the required level $I_{\text {rev }}=I_{\text {ini }}$. Sign of feedback did not depend on the output current polarity because of circuit configuration.

By the way, all the firing pulses of Switches SW1-SW4 are taken away by the Clock pulse front for more reliable switching off of the Bridge-Inverter. The next stage of process of current polarity changing occurs with the next Clock pulse. During this stage SW2 and SW3 became switched off but SW1 and SW4 became switched on. As a result the period of current polarity changing is twice longer than the period of Clock pulses.

One can see that switching off of the Bridge-Inverter Thyristors cause the break of its input current. To avoid the V-1000 output current break the shunting Switch SW5 with resistor R1 is installed in parallel with the Bridge. This Switch comes into the conducting mode at the beginning of the switching process and switches off when BridgeInvertor became conducting. Two transistors (KS621K60 type, Powerex) with driving circuit perform the role of SW5. The Fast Diode D1 and Choke L1 are installed to protect the DCCT-1 against the short pulsed current of switching process and to support this process. DC Current Transformer DCCT-2 is installed at the output bus to measure and to stabilize the Electromagnet current.

PS for the Prototype and for the EMW have closely the 

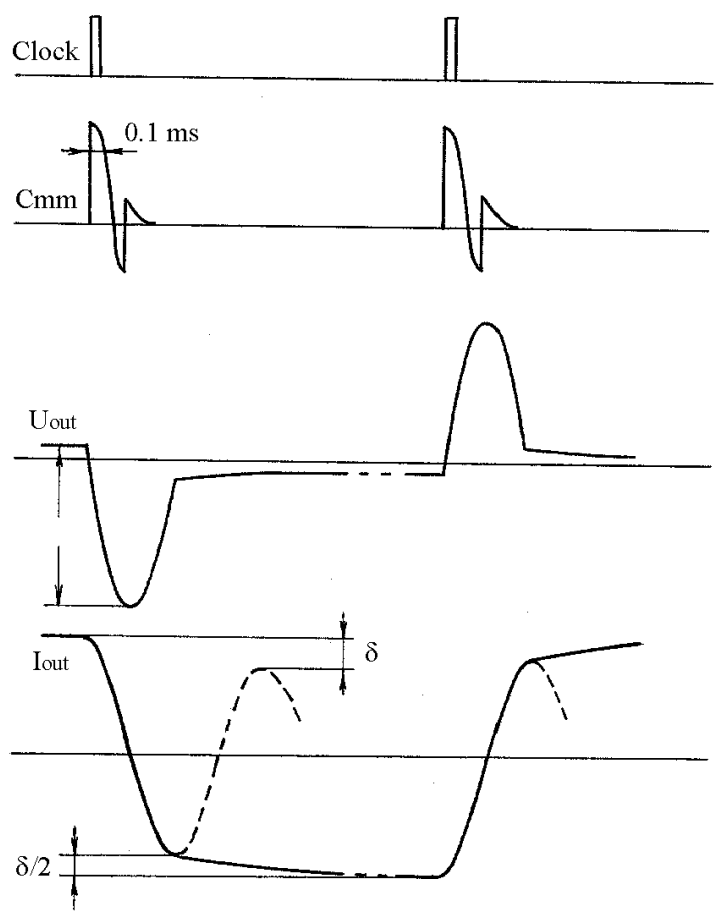

Figure 2: Current switching diagrams.

same frame dimentions: $1200 \mathrm{~mm}$ (front width)x $900 \mathrm{~mm}$ (depth)x $2200 \mathrm{~mm}$ (height).

\section{PECUliarities}

Power Supply for the Prototype and for the EMW have some peculiarities in circuitry, design and operation. First of all, the current stabilizing loop of the EMW is organized with measuring of the current at the bus behind the BridgeInverter (Fig.1), but for the Prototype it was organized with the help of DCCT installed before it. This is determined by the difference in load time constant and in switching frequency. As a result the system for the Prototype is to stabilize the current "in long time mode" only but the feedback loop of the PS for EMW took part in transient processes at the top part of the current and slightly helps to shorten the time duration of its " $\delta / 2$-part". Of course, the feedback loop of the PS for EMW need to re-reverse the DCCT-2 signal polarity (to make it unipolar). Another difference between PSs is in design of the Commutator: we need to apply the recuperation for charging the Capacitor for the Prototype because of high maximal operational frequency and relatively simple circuit with direct capacitor charging - for the EMW. Computer control of the PS for Prototype is organized without special interface module inside PS frame but the frame of the EMW PS includes the AB (Allan-Bradley) Crate. Both systems have the analog and digital status control, of course. Systems were successfully incorporated into the computer control systems of the Storage Rings. Both PSs need the external Clock pulses and they have three types of output pulses to synchronize the external devices like, for example, correcting magnet PS and magnetic field measuring system [3].

\section{RESULTS AND CONCLUSIONS}

The described PS for Prototype had been developed and tested at the 1994. It is under the AC mode of operation with frequencies $2 \mathrm{~Hz}, 10 \mathrm{~Hz}$, and $100 \mathrm{~Hz}$ at the NSLS from January, 1995. The PS for the EMW had been developed, tested and shipped to ANL from INP at May, 1996. EMW with this PS were installed to APS storage ring at December, 1996 and the first experimental runs with the beam were successfully done.

\section{ACKNOWLEDGEMENTS}

Authors are gratefully acknowledged to Drs. Gennady Kulipanov (INP), Efim Gluskin (ANL), and Sam Krinsky (BNL) for so interesting task and for the high attention to this work.

\section{REFERENCES}

[1] A. Fridman, S. Krinsky, E. Blum, K. Halbach, in Proc. of IEEE Part. Acc. Conf., Washington, DC, May, 1993, p. 1599.

[2] E. Gluskin et al., "The elliptical multipole wiggler project", in Proc. of IEEE 1995 Part. Acc. Conf., Dallas, TX, May, 1995, p.1427.

[3] D. Frachon et al.,"Results of Magnetic Measurements and Field Integral compensation for the Elliptical Multipole Wiggler", in Proc. of IEEE 1995 Part. Acc. Conf., Dallas, TX, May, 1995, p.1429. 